\title{
The nature and extent of upper limb associated reactions during walking in people with acquired brain injury
}

\author{
Michelle B. Kahn ${ }^{1,2^{*}}$ (D), Ross A. Clark², Gavin Williams ${ }^{1,3}$, Kelly J. Bower ${ }^{3}$, Megan Banky ${ }^{1,2}$, John Olver ${ }^{4}$ and \\ Benjamin F. Mentiplay ${ }^{5}$
}

\begin{abstract}
Background: Upper limb associated reactions (ARs) are common in people with acquired brain injury (ABI). Despite this, there is no gold-standard outcome measure and no kinematic description of this movement disorder. The aim of this study was to determine the upper limb kinematic variables most frequently affected by ARs in people with $\mathrm{ABI}$ compared with a healthy cohort at matched walking speed intention.

Methods: A convenience sample of 36 healthy control adults (HCs) and 42 people with ABI who had upper limb ARs during walking were recruited and underwent assessment of their self-selected walking speed using the criterionreference three dimensional motion analysis (3DMA) at Epworth Hospital, Melbourne. Shoulder flexion, abduction and rotation, elbow flexion, forearm rotation and wrist flexion were assessed. The mean angle, standard deviation (SD), peak joint angles and total joint angle range of motion (ROM) were calculated for each axis across the gait cycle. On a group level, ANCOVA was used to assess the between-group differences for each upper limb kinematic outcome variable. To quantify abnormality prevalence on an individual participant level, the percentage of ABI participants that were outside of the $95 \%$ confidence interval of the HC sample for each variable were calculated.

Results: There were significant between-group differences for all elbow and shoulder abduction outcome variables $(p<0.01$ ), most shoulder flexion variables (except for shoulder extension peak), forearm rotation SD and ROM and for wrist flexion ROM. Elbow flexion and shoulder abduction were the axes most frequently affected by ARs. Despite the elbow being the most prevalently affected $(38 / 42,90 \%)$, a large proportion of participants had abnormality, defined as $\pm 1.96 \mathrm{SD}$ of the HC mean, present at the shoulder (32/42, 76\%), forearm (20/42, 48\%) and wrist joints (10/42, 24\%).

Conclusion: This study provides valuable information on ARs, and highlights the need for clinical assessment of ARs to include all of the major joints of the upper limb. This may inform the development of a criterion-reference outcome measure or classification system specific to ARs.
\end{abstract}

Keywords: Acquired brain injury, Upper limb, Associated reactions, Three-dimensional motion analysis, Kinematics

\section{Background}

People with acquired brain injury (ABI) often present with movement abnormalities including upper limb associated reactions (ARs) during walking $[1,2]$. Associated reactions are prevalent, recently being reported as a key goal area in $43 \%$ of people in a large stroke cohort $(n=964)$ [3].

\footnotetext{
* Correspondence: Michelle.Kahn@epworth.org.au

'Department of Physiotherapy, Epworth Rehabilitation, Epworth Healthcare,

Melbourne, Australia

${ }^{2}$ School of Health and Sport Sciences, University of Sunshine Coast, Sunshine

Coast, Australia

Full list of author information is available at the end of the article
}

Associated reactions are an effort-dependent phenomenon causing an involuntary increase in upper limb muscle tone, with awkward and uncomfortable postures [4]. Normal arm swing in walking is important to reduce energy expenditure [5], enhance gait stability and balance [6] and facilitate leg swing for faster walking speeds [7-9]. Abnormal upper limb kinematics resulting from ARs may negatively impact gait [10], balance [11], dynamic upper limb function $[12,13]$ and activities of daily living [10] for people with ABI. The treatment of ARs is therefore commonly a focus for physical and pharmacological management $[3,14]$. 
Despite the prevalence and significance of ARs, there are many issues that exist in this field, such as, inconsistent terminology, no gold-standard assessment, unconfirmed contributing factors and varied treatment without supporting evidence [15]. In regards to assessment, there is currently no gold-standard outcome measure, with most having poor ecological validity for walking, involving stationary tests performed in a seated position [4]. The elbow joint is frequently the focus of assessment [4], despite literature suggesting that ARs affect all joints of the upper limb $[16,17]$. Therefore, investigation into the upper limb movement abnormalities caused by ARs during walking is required.

Instrumented three-dimensional motion analysis (3DMA) is the criterion-reference for objective evaluation of joint kinematics during walking [18]. Despite the potential for 3DMA to fulfil the requirements of detailed dynamic upper limb assessment, it is not yet widely integrated into research or clinical practice. To date there have only been a few studies that have developed upper limb marker sets. These have been used for evaluation of arm posture during walking in healthy controls (HCs) [19, 20], paediatric cerebral palsy [21-23] and adults with stroke [2, 24]. While these studies have refined the use of upper body marker sets in gait analysis there has been no research to date in applying 3DMA specifically for the evaluation of ARs. Given that clinically, people with ARs and their therapists often describe ARs in terms of the visual impact, 3DMA is an appropriate methodology to quantify ARs.

A comprehensive assessment of the kinematics of upper limb ARs during walking in ABI may provide insight into the key abnormalities, facilitate the development of a criterion-reference outcome measure, help guide assessment, and clinical decision-making regarding therapeutic interventions. The aim of this study was therefore to determine the upper limb kinematic variables most frequently affected by ARs in people with ABI compared with a healthy cohort.

\section{Methods}

This study was approved by the Human Research Ethics Committees of Epworth Healthcare and the University of the Sunshine Coast (HREC 648-14 and S/17/1006, respectively). All recruitment and testing was performed at Epworth Hospital, Melbourne, Australia. All subjects who were invited, consented to do so and provided written informed consent prior to assessment.

\section{Participants}

\section{ABI participants}

Individuals attending physiotherapy (either in- or outpatient therapy) for mobility limitations after ABI were invited to participate. The inclusion criteria were people who had; 1) an adult-onset ABI (either traumatic brain injury, stroke or stable neurosurgical condition), 2) an $\mathrm{AR}$ in their hemiplegic upper limb during walking as diagnosed by their treating team according to visual observation, 3) $>18$ years of age, 4) could mobilise $10 \mathrm{~m}$ unassisted with no gait aid barefoot, and 5) able to provide consent. Participants were excluded if they had significant cognitive impairment, were unable to follow instructions in English, pregnant, or if they were medically unstable.

\section{Healthy control $(H C)$ participants}

A convenience sample of healthy, injury-free adults were also recruited from the hospital network for comparative purposes [25]. These participants were included if they were more than 18 years of age and had no comorbidities or medical conditions that could impact either of their upper limbs or their capacity to walk (such as a prior neurological condition diagnosis, musculoskeletal injury or severe arthritis).

\section{Procedure}

All participants performed a single testing session, lasting approximately 1 hour. Participants were required to wear tight-fitting shorts and a singlet top (for females) that allowed for placement of the reflective markers. The sample size requirement for the specific ANCOVA model used in this analysis was calculated using $G^{*}$ Power [26]. Inputs of alpha $=0.05$ and power $=0.80$ were used and the ANCOVA model was matched to this study design. The required sample size was calculated based on a prior study examining arm movements in people with stroke [15]. This study reported an effect size of 0.92 , resulting in a required sample size of $n=13$. Because of this very low sample size requirement, the sample size was recalculated with a more modest effect size $(E S=0.50)$. This resulted in a required sample size of $n=34$ participants.

\section{Equipment}

Data collection was performed using a 13-camera Optitrack 3DMA system incorporating 1.3 megapixel cameras sampling at $120 \mathrm{~Hz}$. Motive Body version 1.9 .0 (NaturalPoint, Inc., Corvallis OR, USA) was used to capture the data.

\section{Marker placement}

Small (12.7 $\mathrm{mm}$ diameter) passive reflective markers were mounted directly on the skin with double-sided tape and reinforced with stretch tape. A full body marker set was used, with the markers placed on the participants' upper extremities, forearms, hands, thorax, pelvis, thighs, shanks and feet. Data for this study only used the bilateral upper limb and trunk markers. The 
same assessor, a senior physiotherapist with extensive anatomical training (author MBK), performed all marker placements and captured the data for all testing sessions. A static trial was captured prior to the walking trials to allow for static calibration of the participant to the model. To ensure visibility of all the markers, the participants were instructed to stand still in the anatomical position with their arms away from their body, stretched out as far as possible, their feet shoulder width apart and palms facing forward (as able).

\section{Gait trials}

The participants performed a maximum of six walking trials at a self-selected walking speed along an $8 \mathrm{~m}$ walkway in order to obtain three successful trials. A trial was considered successful where at least one full steady-state gait cycle (i.e. a complete stride) was recorded in the middle of the walkway. Due to the length of the walkway, data for multiple gait cycles were often obtained. The effort-dependent nature of ARs is well-established [27-29] and is inherent in the definition of this phenomenon, whereby ARs occur at initiation of effort and further increase the more effort that is exerted. Although convention dictates that walking speed between $\mathrm{ABI}$ and $\mathrm{HC}$ cohorts are usually matched for comparison, in this particular instance, given that effort is key to ARs, walking speed intention was matched rather than the resultant speed itself. Both the $\mathrm{ABI}$ and $\mathrm{HC}$ participants were therefore matched with the same effortbased instructions so that when they were instructed to walk at their "usual comfortable walking speed, as if they were walking down the street casually" they were exerting the same amount of effort, despite different actual walking speeds. The participants selected their own speeds of walking in response to the given instructions.

\section{Data processing}

All data captured on Motive Body 1.9.0 was cleaned by labelling markers and filling gaps before being imported into Visual3D, professional version 6.01 .15 (C-motion, Inc., Germantown, MD USA), to create the 3D gait model for the upper body. This was performed by the same assessor that performed the participant testing (author M.B.K). The custom upper body model was based on previous research $[20,30]$, with further details provided in the Additional file 1.Marker trajectories were filtered using a $10 \mathrm{~Hz}$, 4th order, zero phase shift lowpass Butterworth filter as performed in previous research [31]. The kinematic data (upper limb joint angles across the gait cycle) were then calculated and exported from Visual3D.

A custom-written software program (LabVIEW National Instruments, Austin TX, USA) was used to analyse the kinematic data across the walking trials. The gait cycle was determined as the time between ground contact of one limb to the subsequent ground-contact of the same limb (defined as the local minimum in the vertical position of the calcaneus marker). As per previously described methods [32], the toe off was defined as the point of maximum displacement from the toe marker to a virtual marker halfway between the two posterior superior iliac spine markers. For the healthy subjects, data were extracted for both the left and right limbs and then averaged for each participant. This was performed because the participants had no pathology, there was no functional relevance of upper limb dominance for gait and as per previous work [33], there was no significant differences between limbs in the kinematics of healthy subjects. Multiple gait cycles were analysed for each of the three successful walking trials. Due to differing walking speeds between the $\mathrm{HC}$ and $\mathrm{ABI}$ cohorts, there were various numbers of gait cycles captured within each walking trial for each participant. Therefore, in order to avoid potential bias in the results where there was differing number of gait cycles, the specific outcomes for each gait cycle were calculated, followed by an average across the available gait cycles for each participant. Additionally, to visually present the data, time normalised average curves were generated for each joint axis and for each participant, and then averaged across each cohort to generate a mean $( \pm 1 \mathrm{SD})$ curve for the $\mathrm{HC}$ and $\mathrm{ABI}$ groups respectively. For the $\mathrm{ABI}$ cohort, only data from each ABI participants' more affected side was included. All waveform data were visualised and inspected for any outliers using custom software. Visual inspection of all walking trials was performed to ensure no erroneous recordings with excessive marker dropout or unexpected results from the model. Any data that was not physiologically plausible or that had excessive missing data were removed prior to the analysis. For visual reporting of the data, gait cycles were time normalised to 101 data points. The six upper limb axes (defined as $+/$ - angular values) of shoulder flexion/extension, shoulder abduction/adduction, shoulder internal/external rotation, elbow flexion/extension, forearm pronation/supination and wrist flexion/extension were analysed. Five outcomes variables were reported for each joint axis, specifically:

1) Mean angle: This reflects the average position of the joint in the particular axis over the gait cycle to give an overall impression of the joint position.

2) Standard deviation of the angle: This reflects the amount of joint axis position variation over the gait cycle and is a measure of variability.

3) Peak joint angle in the positive direction: This reflects the maximum position that the joint 
reaches during the gait cycle in the direction of the axes listed previously (e.g. peak elbow flexion)

4) Peak joint angle in the negative direction: This reflects the peak position that the joint reaches during the gait cycle in the opposite, negative direction to outcome 3 (e.g. peak shoulder extension).

Note: The forearm is typically reported with the anatomical position as the zero reference point: i.e. fully supinated. However, to ensure simpler interpretation the forearm supination values were offset by $90^{\circ}$ to make the zero reference point a neutral position (i.e. midprone), with positive values indicating pronation and negative values indicating supination.

5) Range of motion: This reflects the total excursion of the joint between outcomes 3 and 4, throughout the gait cycle and demonstrates the extent of joint movement.

\section{Statistical analyses}

In order to describe ARs, descriptive summary statistics (mean, SD, range) were used to summarise the shoulder, elbow, forearm and wrist kinematics variables.

Assessment of between-group demographic differences at baseline was performed using independent $t$-tests and revealed significant differences between the $\mathrm{HC}$ and $\mathrm{ABI}$ cohorts. To control for the differences in sex, age, weight, and height, a one-way analysis of covariance (ANCOVA) was performed. ' $F$ ', ' $p$ ' significance value and partial Eta squared values were obtained for each outcome measure comparison to quantify the significance of differences in upper-limb kinematics between the $\mathrm{HC}$ and $\mathrm{ABI}$ participants on a group level, whilst controlling for covariates. Gait velocity was not included as a covariate given that people with $\mathrm{ABI}$ that present with a more severe functional deficit and slower gait speed may in fact present with larger AR joint angles. Therefore, controlling for gait speed could attenuate these outcomes and influence the results unnecessarily.

Whilst the between-group analysis provides important information looking at the features on a group level, for some axes and variables, some of the ABI cohort may deviate in equal proportions in both the positive and negative directions. Between-group analyses would therefore fail to demonstrate the overall abnormality that exists in the cohort on an individual level.

In order to look at the prevalence of abnormality on an individual participant level, results for the individual ABI participants were also compared to the HC sample. The $95 \%$ CIs $( \pm 1.96 \mathrm{SD})$ were calculated for each variable and then each participant classified as normal, increased or decreased for each outcome variable. ABI participants were classified as normal if they fell within the $95 \%$ CIs of the $\mathrm{HC}$ mean for each variable (i.e. mean, standard deviation, peak positive, peak negative and range of motion). Values greater and lower than this range were deemed increased and decreased respectively.

All analyse were performed using Microsoft Excel 2013 and IBM SPSS V24. Where relevant, significance for the $p$ value was set at $<0.05$.

\section{Results \\ Participant demographics}

Forty-two people with ABI and $36 \mathrm{HCs}$ were included in this study, excessing our sample size requirements. Table 1 summarises the demographic data for the ABI and $\mathrm{HC}$ samples and demonstrates the between-group differences at baseline for age, weight, and sex $(p<0.05)$. ANCOVA analysis did not significantly affect the statistical findings.

\section{Between-group differences}

All data were assessed for normality using tests of Skewness and Kurtosis, with the majority of the outcome variables conforming to a normal distribution. The ABI and HC summary statistics for each outcome variable are outlined in Table 2. Figure 1 summarises the average kinematic data for the six upper limb axes for both groups. The ABI cohort was significantly different from the $\mathrm{HCs}$ for 18 out of the 30 variables. All shoulder abduction and elbow flexion outcomes were significantly different between groups $(p<0.01)$.

\section{Abnormality classification at an individual level}

The frequency of $\mathrm{ABI}$ classification beyond the $\mathrm{HC}$ 95\% CI (1.96SD) for the affected upper limb is presented in Table 3. Overall, the ABI cohort walked with significantly more shoulder abduction and elbow flexion. Approximately half the cohort had a more fixed elbow flexion pattern throughout, whereas the remainder had excessive movement moving in and out of elbow flexion. A third had increased shoulder abduction variability. Half the ABI cohort had increased forearm rotation ROM.

For some axes and variables, participants displayed abnormality in both the positive and negative directions. Therefore, there was disparity when looking at the between-group comparisons and classification of abnormality. Shoulder extension peak was not significantly different between the cohorts $(p=0.49)$. However, more than half of the participants were abnormal $(22 / 42,52 \%)$. Nine of these participants had shoulder extension in excess compared to the $\mathrm{HCs}$, whereas 13 were more flexed throughout the gait cycle. Conversely, whilst there was a small but significant difference 
Table 1 Participant demographics

\begin{tabular}{|c|c|c|c|c|c|}
\hline \multirow[t]{2}{*}{ Characteristics } & \multicolumn{2}{|l|}{ Subjects with $\mathrm{ABI}(n=42)$} & \multicolumn{2}{|l|}{$\mathrm{HCs}(n=36)$} & \multirow[t]{2}{*}{$p$} \\
\hline & Range & & Range & & \\
\hline Sex (male/female) & $26 / 16$ & & $13 / 23$ & & 0.02 \\
\hline Age (years) & $48.4 \pm 16.7$ & 20 to 84 & $36.1 \pm 14.8$ & 21 to 78 & $<0.01$ \\
\hline Weight (kg) & $80.03 \pm 16.05$ & 46.9 to 130.5 & $69.3 \pm 12.3$ & 44.9 to 103.1 & $<0.01$ \\
\hline Height (cm) & $172.3 \pm 8.4$ & 155.0 to 190.0 & $169.7 \pm 9.3$ & 149.5 to 187.5 & 0.21 \\
\hline Injury Type (n) & TBI (15), CVA (25), NS (2) & & & & \\
\hline Time Post Injury (years) & $6.2 \pm 5.7$ & 0.2 to 40.4 & & & \\
\hline Gait Velocity (m/s) & $0.85 \pm 0.29$ & 0.11 to 1.51 & $1.27 \pm 0.14$ & 0.96 to 1.51 & $<0.01$ \\
\hline Hemiplegic Side (L/R) & $26 / 16$ & & & & \\
\hline
\end{tabular}

Values are mean \pm SDs unless otherwise indicated

$T B I$ traumatic brain injury, CVA cerebrovascular accidence, NS neurosurgical

between groups for shoulder flexion ROM and SD, on an individual level the ABI cohort overall fell within the 95\% CI. There was no statistical difference between groups for shoulder rotation outcomes, yet, more than a quarter of participants were classified as abnormal for mean $(12 / 42,29 \%)$, shoulder internal rotation peak (13/ $42,31 \%)$ and shoulder external rotation peak (12/42, $29 \%)$. Similar findings were observed for forearm pronation and wrist flexion. For example, there was no group difference for forearm supination peak angle, despite $40 \%$ of participants being classified as abnormal. This was due to near even distribution, with 10 participants more supinated and seven more pronated. There was also no group difference for wrist flexion mean, however $24 \%$ of participants were classified as abnormal, with equal numbers more flexed and extended. This illustrates the importance of examining both group and individual data.

\section{Discussion}

This is the first comprehensive and preliminary description of the nature and extent of kinematic abnormalities of ARs in people with ABI. The results of this study highlight that the key kinematic abnormalities occur predominantly at the elbow and shoulder joints. However, all of the upper limb joint axess may be implicated and there is no single defining feature. Interestingly, despite some between-group differences, total ROM and movement variability (i.e. SD) were within the normal range for each of the joint axes for most ABI participants. This indicates that movement amplitudes and patterns may be somewhat normal in most people with ARs, however they are offset to a different mean position within the joint's ROM. This does not always occur in a typical position, with many axes showing individual participant abnormalities in peak joint position in either direction, not just the mean position. Directional patterns did however exist, with no ABI participants demonstrating more shoulder adduction or elbow extension than the $\mathrm{HC}$ cohort.

To date, the measurement of ARs has focussed primarily on the elbow joint or the muscles acting at the elbow. The systematic review on AR assessment methods by Kahn et al. (2016) [4] demonstrated that eight of the 18 included studies (44\%) only evaluated the AR at the elbow joint, and three (17\%) measured the elbow joint plus one or two additional joints. No study measured all the major joints of the upper limb. Our results do indicate that the elbow is the most frequently affected joint. However, it is essential to highlight that $76 \%$ of people with an ABI presented with abnormal patterns at their shoulder joint, $48 \%$ at the forearm and $24 \%$ at the wrist. This therefore emphasises that comprehensive evaluation of ARs requires inclusion of all the major upper limb joints and should not merely focus on the elbow.

Global AR assessment should be performed in order to guide further impairment-based testing, which in turn should inform targeted treatment. Different abnormalities are likely to require different interventions. A joint that has an average angular position (i.e. mean angle) but is in a fixed position (i.e. low range of motion), versus a joint that has excessive movement (i.e. high range of motion) to a greater maximum angle will require different interventions. For example, a shoulder joint that presents with adduction and internal rotation, with reduced movement (i.e. ROM) and variability (i.e. SD) may indicate the need for a spasticity assessment to determine whether Botulinum Neurotoxin-A injections to shoulder musculature may be beneficial [34]. On the contrary, a shoulder joint with increased shoulder abduction peak, mean and range of motion may indicate the need for a detailed strength and control assessment to inform the prescription of various trunk and shoulder girdle strength and motor control exercises. Additionally, the extent of upper limb joint involvement may assist in differentiating AR movement patterns or levels of 
Table 2 Upper limb kinematic variables matched with self-selected walking speed intention

\begin{tabular}{|c|c|c|c|c|c|c|c|}
\hline \multirow[t]{2}{*}{ Variable $\left(^{\circ}\right)$} & \multicolumn{2}{|l|}{$A B I(n=42)$} & \multicolumn{2}{|l|}{$\mathrm{HC}(\mathrm{n}=36)$} & \multicolumn{3}{|c|}{ ANCOVA OUTCOMES } \\
\hline & Mean \pm SD & Range & Mean \pm SD & Range & $\mathrm{F}$ & $p$ value & Partial Eta Squared \\
\hline Shoulder Flexion Mean & $0.21 \pm 10.58$ & -34.7 to 26.9 & $4.3 \pm 3.3$ & -3.5 to 11.7 & 6.98 & $<0.01$ & 0.09 \\
\hline Shoulder Flexion SD & $4.4 \pm 2.4$ & 0.7 to 10.2 & $8.1 \pm 2.9$ & 3.1 to 18.6 & 24.46 & $<0.01$ & 0.25 \\
\hline Shoulder Flexion Peak & $7.4 \pm 11.1$ & -29.2 to 29.9 & $16.7 \pm 6.3$ & 3.0 to 33.3 & 18.39 & $<0.01$ & 0.20 \\
\hline Shoulder Extension Peak ${ }^{a}$ & $-7.3 \pm 11.2$ & -39.3 to 23.2 & $-7.7 \pm 4.2$ & -21.4 to -1.2 & 0.48 & 0.49 & 0.01 \\
\hline Shoulder Flexion ROM & $14.7 \pm 7.1$ & 3.3 to 30.4 & $24.5 \pm 8.5$ & 10.8 to 54.6 & 18.18 & $<0.01$ & 0.20 \\
\hline Shoulder Abduction Mean & $16.9 \pm 7.7$ & 3.7 to 36.2 & $6.4 \pm 3.1$ & 0.6 to 12.8 & 38.34 & $<0.01$ & 0.35 \\
\hline Shoulder Abduction SD & $2.6 \pm 1.3$ & 0.4 to 6.2 & $1.8 \pm 0.6$ & 0.8 to 3.2 & 12.08 & $<0.01$ & 0.14 \\
\hline Shoulder Abduction Peak & $21.3 \pm 7.9$ & 7.0 to 37.9 & $9.4 \pm 3.3$ & 2.5 to 16.5 & 48.49 & $<0.01$ & 0.40 \\
\hline Shoulder Adduction Peak & $12.9 \pm 8.1$ & -1.27 to 34.7 & $3.7 \pm 3.2$ & -2.7 to 10.4 & 23.26 & $<0.01$ & 0.24 \\
\hline Shoulder Abduction ROM & $8.5 \pm 4.0$ & 1.6 to 20.6 & $5.8 \pm 1.8$ & 3.1 to 9.6 & 15.91 & $<0.01$ & 0.18 \\
\hline Shoulder Rotation Mean & $16.7 \pm 20.6$ & -24.4 to 62.3 & $10.3 \pm 12.1$ & -10.9 to 34.4 & 0.40 & 0.53 & 0.01 \\
\hline Shoulder Rotation SD & $4.0 \pm 1.9$ & 1.4 to 9.6 & $4.5 \pm 2.2$ & 2.0 to- 12.0 & 0.01 & 0.92 & 0.00 \\
\hline Shoulder Internal Rotation Peak & $24.4 \pm 20.5$ & -19.4 to 68.3 & $16.8 \pm 12.0$ & -2.6 to 40.3 & 1.18 & 0.28 & 0.02 \\
\hline Shoulder External Rotation Peak & $9.5 \pm 21.1$ & -37.1 to 57.5 & $1.9 \pm 13.7$ & -23.3 to 27.9 & 0.39 & 0.54 & 0.01 \\
\hline Shoulder Rotation ROM & $14.9 \pm 6.7$ & 5.7 to 30.2 & $14.9 \pm 6.4$ & 6.7 to 36.5 & 1.19 & 0.28 & 0.02 \\
\hline Elbow Flexion Mean & $50.7 \pm 24.1$ & 14.3 to 109.9 & $11.9 \pm 6.0$ & -0.6 to 26.1 & 63.52 & $<0.01$ & 0.47 \\
\hline Elbow Flexion SD & $3.4 \pm 1.5$ & 0.9 to 8.8 & $9.6 \pm 3.4$ & 3.1 to 17.0 & 80.04 & $<0.01$ & 0.53 \\
\hline Elbow Flexion Peak & $56.6 \pm 23.3$ & 20.8 to 115.9 & $27.4 \pm 7.9$ & 12.2 to 43.7 & 36.14 & $<0.01$ & 0.33 \\
\hline Elbow Extension Peak ${ }^{\mathrm{a}}$ & $44.5 \pm 24.8$ & 8.9 to 103.4 & $-0.6 \pm 7.9$ & -14.3 to 17.7 & 79.80 & $<0.01$ & 0.53 \\
\hline Elbow Flexion ROM & $12.0 \pm 5.0$ & 4.1 to 26.8 & $28.1 \pm 9.1$ & 10.3 to 46.1 & 66.23 & $<0.01$ & 0.48 \\
\hline Forearm Rotation Mean ${ }^{b}$ & $16.6 \pm 27.8$ & -62.8 to 67.9 & $17.4 \pm 13.7$ & -6.1 to 52.9 & 0.18 & 0.67 & 0.00 \\
\hline Forearm Rotation SD ${ }^{\mathbf{b}}$ & $-84.7 \pm 3.4$ & -89.0 to -71.4 & $-86.3 \pm 1.3$ & -88.2 to -83.3 & 12.89 & $<0.01$ & 0.15 \\
\hline Forearm Pronation Peak ${ }^{b}$ & $27.0 \pm 29.0$ & -51.5 to 82.7 & $24.2 \pm 14.9$ & -2.2 to 63.5 & 1.58 & 0.21 & 0.02 \\
\hline Forearm Supination Peak & $6.3 \pm 27.5$ & -73.6 to 54.6 & $11.3 \pm 13.1$ & -10.8 to 43.3 & 0.32 & 0.57 & 0.00 \\
\hline Forearm Rotation ROM ${ }^{\mathbf{b}}$ & $20.7 \pm 13.0$ & 4.3 to 69.7 & $12.9 \pm 3.9$ & 6.8 to 21.2 & 19.34 & $<0.01$ & 0.21 \\
\hline Wrist Flexion Mean & $-5.8 \pm 12.5$ & -41.5 to 33.4 & $-5.6 \pm 6.4$ & -20.6 to 9.1 & 0.04 & 0.84 & 0.00 \\
\hline Wrist Flexion SD & $2.4 \pm 1.6$ & 0.5 to 7.0 & $2.5 \pm 1.0$ & 1.0 to 4.7 & 1.43 & 0.24 & 0.02 \\
\hline Wrist Flexion Peak & $-0.9 \pm 13.3$ & -37.0 to 34.8 & $-1.0 \pm 7.0$ & - 13.7 to 19.3 & 0.40 & 0.53 & 0.01 \\
\hline Wrist Extension Peak ${ }^{a}$ & $-10.2 \pm 12.6$ & -44.5 to 32.36 & $-9.6 \pm 6.6$ & -26.7 to 4.0 & 0.13 & 0.72 & 0.00 \\
\hline Wrist Flexion ROM & $9.3 \pm 6.3$ & 1.9 to 25.6 & $8.5 \pm 3.2$ & 3.8 to 16.1 & 5.39 & $<0.05$ & 0.07 \\
\hline
\end{tabular}

All units for mean \pm SD and range are in degrees and for the affected upper limb. The bold variables are significantly different between groups $A B I$ acquired brain injury, $H C$ healthy control, $S D$ standard deviation, $R O M$ range of motion

${ }^{a}$ values for these axis vectors are higher the lower the value is. For these variables 0 represents the neutral position, and a more negative value denotes a greater angle. For example, the mean Forearm Supination Peak for the $\mathrm{ABI}$ group was $6.3^{\circ}$, which indicates that the mean angle was still in a pronated position as it was $>0^{\circ}$. By contrast, mean Shoulder Extension Peak for the $A B I$ group was $-7.3^{\circ}$, indicating that the shoulder was in an extended position as it was $<0^{\circ}$. Joint angles are expressed in this manner for clinical clarity

${ }^{\mathrm{b}}$ The forearm is typically reported with the healthy anatomical position as the zero reference point: i.e. fully supinated. By contrast, it is reported here as offset by $90^{\circ}$ to make the zero reference point a neutral position, with positive values more pronated and negative values more supinated

severity. For example, lower limb severity during gait is determined by the number of joints involved and the extent of abnormality at each joint [35, 36], therefore the presence of abnormality at multiple levels of the upper limb may similarly be a determinant of AR severity. Assessment of the elbow in isolation or basic interpretation of joint abnormality could result in contributing factors to a patient's presentation being overlooked, with the patient failing to benefit from potentially treatable problems.
In the lower limb, 3DMA during gait has ultimately led to the development of classification systems and comprehensive treatment algorithms in various neurological populations [37, 38]. Such systems do not exist for upper limb kinematics and postures during gait. There is one upper limb classification system that outlines five upper limb postures in people with upper limb spasticity due to ABI [39]. Hefter et al. (2012) [39] developed this system using expert consensus of visual 


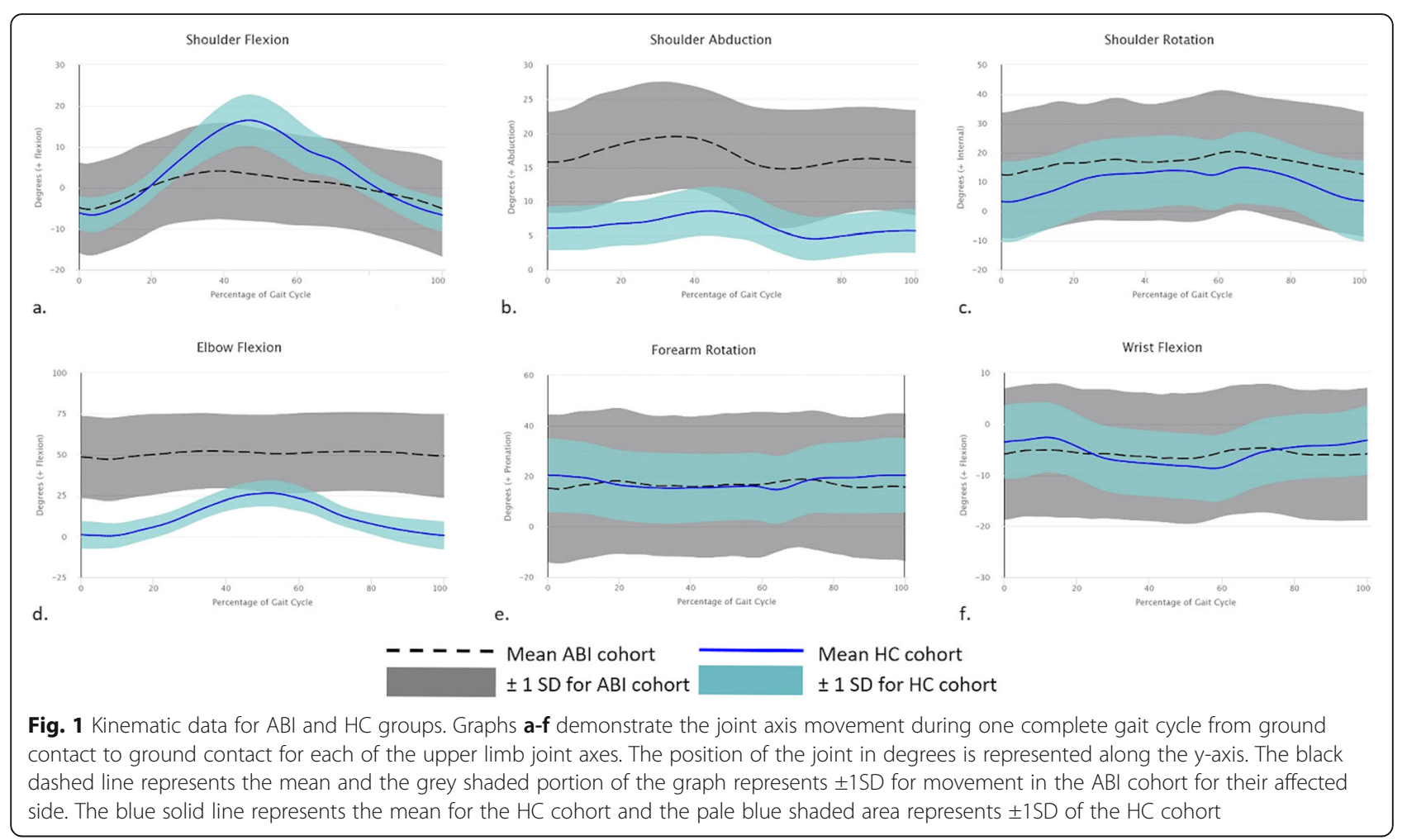

observation during sitting and standing. This panel devised five typical patterns of upper limb posturing. The majority of the upper limb postures involved positions of shoulder internal rotation and adduction and elbow flexion. Other posture differences resulted from the positions of the forearm and wrist. The use of such a classification system would be beneficial to improve therapist communication, facilitate assessment, develop treatment algorithms specific to homogenous subgroups of patients and to prognosticate outcomes. However, the limitations of visual observation in neurological cohorts is well established [40-42].

Although we studied ARs during walking, whereas Hefter et al. [39] did so in sitting and standing, our data do not support the empirical classification proposed. This may be as a result of its development through visual observation, or because of the assessment of a different functional task. The internal shoulder rotation pattern present in all five upper limb categories proposed by Hefter et al. [39] was only present in eight of the 42 participants in this present study (19\%). No participant demonstrated shoulder adduction as evident in patterns I - IV. Our participants were either classified as having increased shoulder abduction or normal abduction/adduction angles. Further, the most common pattern proposed by Hefter et al. [39] (pattern III - 41.8\%), did not exist in our cohort. We found eight participants with the first feature (shoulder internal rotation), but none had shoulder adduction. Of those that had shoulder internal rotation, they all also had elbow flexion. Of those eight people, only one person had a neutral forearm position, and that person also had a neutral wrist. Even without shoulder adduction included, only one of our ABI participants (2.4\%) fitted pattern III. This pattern described a neutral forearm and wrist, neither of which actually occur in walking in healthy adults. The forearm in our HCs was on average $\sim 17^{\circ}$ pronated and the wrist in $\sim 6^{\circ}$ of extension. Visual observation of forearm position may be influenced by shoulder position or lack of clarity in the transverse plane and therefore may not be accurate. We need to rely on accurate data and classification systems that are objectively developed if it is to facilitate treatment decisions.

The inability of the proposed classification system to be applied to our cohort highlights the need for such systems to be specifically evaluated using motion analysis to ensure their accuracy, if the intention is for them to prognosticate outcomes and inform therapeutic management. The disparity between the upper limb kinematics and postures in our cohort during walking and the stationary posture classifications described by Hefter et al. (2012) [39] also further supports the notion that assessment of ARs needs to be dynamic and functional to ensure it is ecologically valid. This research may facilitate the future development of a dynamic, criterionreference outcome measure for accurate and ecologically valid testing of ARs and the development of a classification system specific to ARs of the upper limb. 
Table 3 Incidence of upper limb variable abnormalities (for the affected upper limb with AR)

\begin{tabular}{|c|c|c|c|}
\hline Variable & Decreased n (\%) & Normal n (\%) & Increased n (\%) \\
\hline Shoulder Flexion Mean & $18(43)$ & $18(43)$ & $6(14)$ \\
\hline Shoulder Flexion SD & $9(21)$ & $33(79)$ & $0(0)$ \\
\hline Shoulder Flexion Peak & $17(41)$ & $24(57)$ & $1(2)$ \\
\hline Shoulder Extension Peak & $9(21)$ & $20(48)$ & $13(31)$ \\
\hline Shoulder Flexion ROM & $6(14)$ & $36(86)$ & $0(0)$ \\
\hline Shoulder Abduction Mean & $0(0)$ & $11(26)$ & $31(74)$ \\
\hline Shoulder Abduction SD & $1(2)$ & $28(67)$ & $13(31)$ \\
\hline Shoulder Abduction Peak & $0(0)$ & $10(24)$ & $32(76)$ \\
\hline Shoulder Adduction Peak & $26(62)$ & $16(38)$ & $0(0)$ \\
\hline Shoulder Abduction ROM & $1(2)$ & $26(62)$ & $15(36)$ \\
\hline Shoulder Rotation Mean & $4(10)$ & $30(71)$ & $8(19)$ \\
\hline Shoulder Rotation SD & $0(0)$ & $41(98)$ & $1(2)$ \\
\hline Shoulder Internal Rotation Peak & $4(10)$ & $29(69)$ & $9(21)$ \\
\hline Shoulder External Rotation Peak & $8(19)$ & $30(71)$ & $4(10)$ \\
\hline Shoulder Rotation ROM & $0(0)$ & $39(93)$ & $3(7)$ \\
\hline Elbow Flexion Mean & $0(0)$ & $4(10)$ & $38(91)$ \\
\hline Elbow Flexion SD & $20(48)$ & $22(52)$ & $0(0)$ \\
\hline Elbow Flexion Peak & $0(0)$ & $14(33)$ & $28(67)$ \\
\hline Elbow Extension Peak & $38(91)$ & $4(10)$ & $0(0)$ \\
\hline Elbow Flexion ROM & $17(41)$ & $25(60)$ & $0(0)$ \\
\hline Forearm Rotation Mean & $5(12)$ & $29(69)$ & $8(19)$ \\
\hline Forearm Rotation SD & $1(2)$ & $27(64)$ & $14(33)$ \\
\hline Forearm Pronation Peak & $5(12)$ & $27(64)$ & $10(24)$ \\
\hline Forearm Supination Peak & $7(17)$ & $25(60)$ & $10(24)$ \\
\hline Forearm Rotation ROM & $1(2)$ & $22(52)$ & $19(45)$ \\
\hline Wrist Flexion Mean & $5(12)$ & $32(76)$ & $5(12)$ \\
\hline Wrist Flexion SD & $2(5)$ & $35(83)$ & $5(12)$ \\
\hline Wrist Flexion Peak & $5(12)$ & $33(79)$ & $4(10)$ \\
\hline Wrist Extension Peak & $3(7)$ & $34(81)$ & $5(12)$ \\
\hline Wrist Flexion ROM & $2(5)$ & $32(76)$ & $8(19)$ \\
\hline
\end{tabular}

$S D$ standard deviation, Max maximum, Min minimum, $R O M$ range of motion

The bold variables highlight where the majority of the $A B I$ cohort were classified

\section{Study limitations}

It is usual practice in gait research to match the clinical population and $\mathrm{HC}$ walking speeds to account for the speed-related impact on kinematics [9]. However, as outlined in the methodology, in this particular case, given the effort-dependent nature of ARs, it was more important to match the groups for walking effort or intent. Effort is key to the definition of ARs whereby the greater the effort, the more significant the AR [27-29]. Therefore, this is a more valid comparison. Additionally, gait velocity was not incorporated as a covariate in our analyses for a number of reasons. Firstly, given that people with poorer functional ability and slower gait speeds may have greater ARs angles, controlling for gait velocity could potentially influence and attenuate the AR kinematics obtained. Secondly, none of the outcomes for each of the six upper limb axes were correlated more than moderately to gait velocity $(r<0.5)$.

Another limitation was that this research explores ARs only during walking. This is just one example of a functional activity during which ARs may affect people with ABI. Therefore, the results of this study may not translate to other functional tasks such as sit-to-stand or standing.

Three-dimensional motion analysis is not without limitations. The shoulder joint is complex to model, as it consists of both scapulothoracic and glenohumeral 
components but the model used describes humeral movement relative to the trunk [30]. Additionally, the joint rotation centre is a rough estimate for the ball and socket joint [43] and there may be potential error related to soft tissue artefact [44]. Further, we could not capture data on the fingers. This is an application for future research given that a clenched fist with finger flexion is a common pattern observed in people with upper limb spasticity [39] and is frequently rated by clinicians as being implicated in ARs [16]. However, 3DMA is the criterion-reference system available for biomechanical analysis and therefore provides the most accurate data possible without resorting to complex dynamic imaging systems.

The final limitation is the inclusion of a mixed ABI cohort including stroke, traumatic brain injury and stable neurosurgical conditions and relatively small sample size. Whilst the mixed cohort limits the ability to subgroup the patients based on diagnosis, this is in line with the International Consensus Statement for the management of upper limb disorders of tone [45]. Additionally, the cohort includes only people with ARs as a result of a stable upper motor neurone lesion and excludes other neurological diagnosis such as multiple sclerosis, Parkinson's disease or incomplete spinal cord injury, where the pathophysiology of ARs may be different and the condition may be progressive. Whilst this study has relatively low participant numbers, it is one of the largest studies on ARs published to date and provides a preliminary insight into the kinematics of ARs. A larger sample may determine if subgroups with distinct patterns exist. However, subgrouping by aetiology (between aetiologies), or by pattern (within aetiologies) is beyond the scope of this paper.

\section{Conclusion}

In people with $\mathrm{ABI}$ who presented with ARs during walking, the most frequently affected axes of movement were elbow flexion, shoulder abduction and shoulder flexion. Shoulder rotation, forearm rotation and wrist flexion were implicated to a lesser extent, but were still of importance. These results can help clinicians prioritise their attention during assessment of ARs. Laboratory or clinical evaluation of ARs requires inclusion of all the major joints of the upper limb and not just the elbow joint.

\section{Supplementary information}

Supplementary information accompanies this paper at https://doi.org/10. 1186/s12984-019-0637-2.

Additional file 1. Description of the three-dimensional upper limb model.

\section{Abbreviations}

3DMA: Three-dimensional motion analysis; ABI: Acquired brain injury; ARs: Associated reactions; HCs: Healthy controls; ROM: Range of motion; SD: Standard deviation

\section{Acknowledgements}

Not applicable.

\section{Authors' contributions}

All authors have read and agreed the Statement for Authors. Primary Author M.B.K performed all data collection, data cleaning, data analysis, data interpretation and drafting the manuscript; B.F.M assisted in data collection of the clinical cohort, developed the upper limb model and analysis programs, performed the data processing and contributed to critical revision of the work. G.W and R.C contributed to data analysis, data interpretation and critical revision of the work. M.B assisted in the data collection of the healthy cohort. K.J.B, P.Y.H and J.O contributed to critical revision of the work.

\section{Funding}

This research was supported by grants awarded to Michelle Kahn from the Royal Automobile Club of Victoria (RACV), Epworth Research Institute (ERI) and Physiotherapy Research Fund (PRF). The funds awarded from RACV and ERI were utilised as part of a larger scale research program investigating various outcome measures for gait, spasticity and associated reactions in people with acquired brain injury. Dr. Ross Clark is supported by a National Health and Medical Research Council R.D. Wright Biomedical Fellowship (Number: 1090415).

\section{Availability of data and materials}

The datasets used and/or analysed during the current study are available from the corresponding author on reasonable request.

\section{Ethics approval and consent to participate}

This research is approved by the Human Research Ethics Committees of Epworth Healthcare (HREC 648-14) and the University of the Sunshine Coast $(S / 17 / 1006)$. All subjects that were invited, consented to do so and provided written informed consent prior to assessment.

\section{Consent for publication}

Not applicable.

\section{Competing interests}

The author(s) declared no potential conflicts of interest with respect to the research, authorship and/or publication of this article.

\section{Author details}

'Department of Physiotherapy, Epworth Rehabilitation, Epworth Healthcare, Melbourne, Australia. ${ }^{2}$ School of Health and Sport Sciences, University of Sunshine Coast, Sunshine Coast, Australia. ${ }^{3}$ School of Physiotherapy, The University of Melbourne, Melbourne, Australia. ${ }^{4}$ Epworth Monash Rehabilitation Unit (EMReM), Melbourne, Australia. ${ }^{5}$ La Trobe Sport and Exercise Medicine Research Centre, La Trobe University, Bundoora, Australia.

Received: 26 August 2019 Accepted: 13 December 2019

Published online: 27 December 2019

\section{References}

1. Williams G, Morris ME, Schache A, McCrory PR. Incidence of gait abnormalities after traumatic brain injury. Arch Phys Med Rehabil. 2009; 90(4):587-93.

2. Carmo AA, Kleiner AFR, Lobo da Costa PH, Barros RML. Three-dimensional kinematic analysis of upper and lower limb motion during gait of poststroke patients. Braz J Med Biol Res. 2012;45(6):537-45.

3. Bakheit AM, Zakine B, Maisonobe $\mathrm{P}$, Aymard C, Fhedoroff $\mathrm{K}$, Hefter $\mathrm{H}$, et al. The profile of patients and current practice of treatment of upper limb muscle spasticity with botulinum toxin type a: an international survey. Int J Rehabil Res. 2010;33(3):199-204.

4. Kahn MB, Mentiplay BF, Clark RA, Bower KJ, Williams G. Methods of assessing associated reactions of the upper limb in stroke and traumatic brain injury: a systematic review. Brain Inj. 2016;30(3):252-66.

5. Umberger BR. Effects of suppressing arm swing on kinematics, kinetics, and energetics of human walking. J Biomech. 2008;41(11):2575-80.

6. Ortega JD, Fehlman LA, Farley CT. Effects of aging and arm swing on the metabolic cost of stability in human walking. J Biomech. 2008:41(16):3303-8. 
7. Meyns P, Bruijn S, Duysens J. The how and why of arm swing during human walking. Gait Posture. 2013;38(4):555-62.

8. Stephenson JL, Lamontagne A, De Serres SJ. The coordination of upper and lower limb movements during gait in healthy and stroke individuals. Gait Posture. 2009;29(1):11-6.

9. Romkes J, Bracht-Schweizer K. The effects of walking speed on upper body kinematics during gait in healthy subjects. Gait Posture. 2017;54:304-10.

10. Bhakta BB, O'Connor RJ, Cozens JA. Associated reactions after stroke: a randomized controlled trial of the effect of botulinum toxin type A. J Rehabil Med. 2008;40(1):36-41.

11. Davies PM. Steps to follow: the comprehensive treatment of patients with hemiplegia. Berlin: Springer; 2000.

12. Davies BL, Kurz MJ. Children with cerebral palsy have greater stochastic features present in the variability of their gait kinematics. Res Dev Disabil. 2013:34(11):3648-53

13. Bobath B. Adult hemiplegia: evaluation and treatment. London: Butterworth-Heinemann; 1990.

14. Ashford S, Fheodoroff K, Jacinto J, Turner-Stokes L. Common goal areas in the treatment of upper limb spasticity: a multicentre analysis. Clin Rehabil. 2016;30(6):617-22.

15. Kahn M, Williams $G$. Associated reactions in people with brain injury-a common but poorly defined problem. Int J Ther Rehabil. 2016;23(3):106-7.

16. Kahn MB, Ross CA, Bower KJ, Mentiplay BF, Pua YH, Olver J, Williams G. The reproducibility and responsiveness of subjective assessment of upper limb associated reactions in people with acquired brain injury during walking. Clin Rehabil. 2019. https://doi.org/10.1177/0269215519888782.

17. Brunnstrom S. Associated reactions of the upper extremity in adult patients with hemiplegia; an approach to training. Phys Ther Rev. 1956;36(4):225.

18. Simon SR. Quantification of human motion: gait analysis-benefits and limitations to its application to clinical problems. J Biomech. 2004;37(12) 1869-80.

19. Cimolin V, Galli M, Albertini G, Crivellini M, Romkes J, Brunner R. Quantitative analysis of upper limbs during gait: a marker set protocol. J Appl Biomater Funct Mater. 2012;10(1):49-55.

20. Frykberg GE, Johansson GM, Schelin L, Häger CK. The arm posture score for assessing arm swing during gait: an evaluation of adding rotational components and the effect of different gait speeds. Gait Posture. 2014;40: 64-9.

21. Riad J, Coleman S, Lundh D, Broström E. Arm posture score and arm movement during walking: a comprehensive assessment in spastic hemiplegic cerebral palsy. Gait Posture. 2011;33(1):48-53.

22. Lundh D, Coleman S, Riad J. Movement deviation and asymmetry assessment with three dimensional gait analysis of both upper-and lower extremity results in four different clinical relevant subgroups in unilateral cerebral palsy. Clin Biomech. 2014;29(4):381-6.

23. Romkes J, Peeters W, Oosterom AM, Molenaar S, Bakels I, Brunner R. Evaluating upper body movements during gait in healthy children and children with diplegic cerebral palsy. J Pediatr Orthop B. 2007;16(3):175-80

24. Johansson GM, Frykberg GE, Grip H, Broström EW, Häger CK. Assessment of arm movements during gait in stroke-the arm posture score. Gait Posture. 2014;40(4):549-55.28

25. Mentiplay BF, Banky M, Clark RA, Kahn MB, Williams G. Lower limb angular velocity during walking at various speeds. Gait Posture. 2018;65:190-6.

26. Faul F, Erdfelder E, Lang AG, Buchner A. G* power 3: a flexible statistical power analysis program for the social, behavioral, and biomedical sciences. Behav Res Methods. 2007;39(2):175-91.

27. Dvir Z, Penturin E, Prop I. The effect of graded effort on the severity of associated reactions in hemiplegic patients. Clin Rehabil. 1996;10(2):155-8.

28. Bhakta BB, Cozens JA, Chamberlain MA, Bamford JM. Quantifying associated reactions in the paretic arm in stroke and their relationship to spasticity. Clin Rehabil. 2001;15(2):195-206.

29. Lazarus JC. Associated movement in hemiplegia: the effects of force exerted, limb usage and inhibitory training. Arch Phys Med Rehabil. 1992; 73(11):1044

30. Rab G, Petuskey K, Bagley A. A method for determination of upper extremity kinematics. Gait Posture. 2002;15(2):113-9.

31. Mentiplay BF, Clark RA. Modified conventional gait model versus cluster tracking: test-retest reliability, agreement and impact of inverse kinematics with joint constraints on kinematic and kinetic data. Gait Posture. 2018;64: 75-83.
32. Zeni J Jr, Richards J, Higginson J. Two simple methods for determining gait events during treadmill and overground walking using kinematic data. Gait Posture. 2008;27(4):710-4.

33. Galli M, Cimolin V, Albertini G, Piccinini L, Turconi AC, Romkes J, et al. Kinematic analysis of upper limb during walking in diplegic children with Cerebral palsy. Eur J Paediatr Neurol. 2014;18(2):134-9.

34. Royal College of Physicians, British Society of Rehabilitation Medicine, The Chartered Society of Physiotherapy, Association of Chartered Physiotherapists in Neurology and the Royal College of Occupational Therapists. Spasticity in adults: management using botulinum toxin. National Guidelines. London: Royal College of Physicians; 2018.

35. Schwartz MH, Rozumalski A. The gait deviation index: a new comprehensive index of gait pathology. Gait Posture. 2008;28(3):351-7.

36. Baker R, McGinley JL, Schwartz MH, Beynon S, Rozumalski A, Graham HK, et al. The gait profile score and movement analysis profile. Gait Posture. 2009;30(3):265-9.

37. Rodda J, Graham H. Classification of gait patterns in spastic hemiplegia and spastic diplegia: a basis for a management algorithm. Eur J Neurol. 2001;8: 98-108.

38. Williams G, Lai D, Schache A, Morris ME. Classification of gait disorders following traumatic brain injury. J Head Trauma Rehabil. 2015;30(2):E13-23.

39. Hefter $\mathrm{H}$, Jost WH, Reissig A, Zakine B, Bakheit AM, Wissel J. Classification of posture in poststroke upper limb spasticity: a potential decision tool for botulinum toxin a treatment? Int J Rehabil Res. 2012;35(3):227-33.

40. Williams G, Morris ME, Schache A, McCrory P. Observational gait analysis in traumatic brain injury: accuracy of clinical judgment. Gait Posture. 2009; 29(3):454-9.

41. Watelain E, Froger J, Barbier F, Lensel G, Rousseaux M, Lepoutre FX, et al. Comparison of clinical gait analysis strategies by French neurologists, physiatrists and physiotherapists. J Rehabil Med. 2003;35(1):8-14.

42. Maathuis KGB, van der Schans C, van Iperen A, Rietman J, Geertzen J. Gait in children with cerebral palsy: observer reliability of physician rating scale and Edinburgh visual gait analysis interval testing scale. J Pediatr Orthop. 2005; 25(3):268-72.

43. Wu G. ISB recommendation on definitions of joint coordinate systems of various joints for the reporting of human joint motion--part II: shoulder, elbow, wrist and hand. J Biomech. 2005:38(5):981.

44. Baker R. Gait analysis methods in rehabilitation. J Neuroeng Rehabil. 2006; 3(1):4

45. Sheean G, Lannin NA, Turner-Stokes L, Rawicki B, Snow BJ, Cerebral PI. Botulinum toxin assessment, intervention and after-care for upper limb hypertonicity in adults: international consensus statement. Eur J Neurol. 2010;17(Suppl 2):74-93.

\section{Publisher's Note}

Springer Nature remains neutral with regard to jurisdictional claims in published maps and institutional affiliations.

Ready to submit your research? Choose BMC and benefit from:

- fast, convenient online submission

- thorough peer review by experienced researchers in your field

- rapid publication on acceptance

- support for research data, including large and complex data types

- gold Open Access which fosters wider collaboration and increased citations

- maximum visibility for your research: over $100 \mathrm{M}$ website views per year

At $\mathrm{BMC}$, research is always in progress.

Learn more biomedcentral.com/submissions 